\title{
Effect of intense pulsed light on hydrothermally grown $\mathrm{ZnO}$ nanowires
}

\author{
Abdulaziz K. Assaifan, Naif A. Alshehri, Aled R. Lewis, Siamak Samavat, Y.C. Lau, Davide \\ Deganello and Kar Seng Teng* \\ College of Engineering, Swansea University, Bay Campus, Fabian Way, Crymlyn Burrows, Swansea SA1 8EN, United Kingdom. \\ Corresponding Author Email: K.S.Teng@swansea.ac.uk
}

\begin{abstract}
Hydrothermal growth of $\mathrm{ZnO}$ nanowires has been of great interest as it allows the use of organic substrates for flexible electronics applications. However, there are drawbacks on the growth technique as it is known to introduce defects in the nanowires. In this work, the use of intense pulsed light on hydrothermally grown $\mathrm{ZnO}$ nanowires on polyimide substrate was performed to enhance the physical and electronic properties of the nanomaterials. Such photonic annealing technique offers a rapid and effective improvement in material properties and importantly it is suitable for use on a wide variety of organic substrates, which are vital for flexible electronics.
\end{abstract}

Keywords: photonic annealing; ZnO nanowires; flexographic printing; defects

\section{Introduction}

Hydrothermal growth technique, which can be performed at a temperature of less than $100{ }^{\circ} \mathrm{C}$, is suitable for low-cost mass production on organic substrates. However, it is known to produce nanowires with high density of defects that can influence the electrical and optical properties of the materials [1]. Therefore, post-synthesis treatment such as thermal annealing of $\mathrm{ZnO}$ nanowires is important to improve its properties. However, thermal annealing is time consuming and is unsuitable for organic substrates with low glass transition temperature. 
Laser annealing was previously reported on $\mathrm{ZnO}$ nanowires, which enhanced the crystallinity of the material at much shorter time [2]. However, laser annealing is complex and unviable for high throughput production of nanowires device due to its small spot size.

Photonic annealing has previously been used to sinter printed metallic nanoparticles and to enhance the electrical properties of printed graphene [3, 4]. In this work, the use of intense pulsed light to anneal hydrothermally grown $\mathrm{ZnO}$ nanowires on printed silver on polyimide substrate is reported for the first time. Such photonic annealing technique would anneal the nanomaterials at high temperature without causing damage to the underlying substrates [5]. It offers significant advantages over other techniques as it allows the use of low-cost organic substrates and provides rapid annealing.

\section{Experimental Details}

\subsection{Materials}

Polyimide substrates were obtained from Lohmann Adhesive Tape Systems. Silver ink (PFI 722) was purchased from NovaCentrix. Zinc acetate (ZnA) precursor solution consisted of isopropanol (IPA) (Fisher scientific) and zinc acetate dihydrate (Sigma Aldrich). Zinc nitrate hexahydrate and hexamethylenetetramine (HMTA) (Sigma Aldrich) were used in the hydrothermal growth of $\mathrm{ZnO}$ nanowires. Extra pure DI water and phosphate buffered silane (PBS) solution were purchased from Fisher.

\subsection{Synthesis of $\mathrm{ZnO}$ nanowires}

Flexographic printing technique (IGT Reprotest Printability Tester F1) was used to print silver electrodes and $\mathrm{ZnA}$ on polyimide substrate as reported in previous work [6]. After printing $\mathrm{ZnA}$, the substrate was placed on a hotplate at $350{ }^{\circ} \mathrm{C}$ to allow the conversion of $\mathrm{ZnA}$ to $\mathrm{ZnO}$ seed layers suitable for hydrothermal growth of $\mathrm{ZnO}$ nanowires [6]. The nanowires 
were also grown on silicon substrate in order to measure its length using cross-sectional scanning electron microscope (SEM).

\subsection{Photonic annealing of $\mathrm{ZnO}$ nanowires}

Photonic annealing of $\mathrm{ZnO}$ nanowires was performed using PulseForge 1200 (Novacentrix, USA). The sample was placed at a distance of $20 \mathrm{~mm}$ away from the xenon lamp (wavelength ranging from 200 to $1500 \mathrm{~nm}$ ) and exposed to nitrogen during photonic annealing, which was performed at $350 \mathrm{~V}$ with a pulse envelope of $6.9 \mathrm{~ms}$ (comprising of three identical micro-pulses at a duty cycle of $95.65 \%$ ) to deliver an energy density of $7.93 \mathrm{~J} \mathrm{~cm}^{-2}$. Figure 1 illustrates the fabrication and photonic annealing process of $\mathrm{ZnO}$ nanowires.

(a)

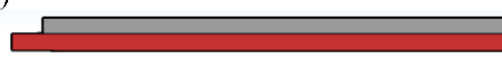

(c)

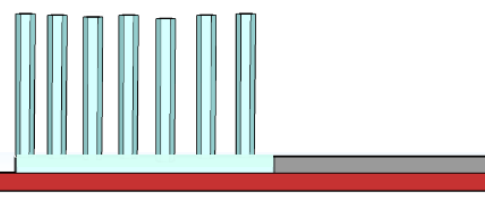

(b)

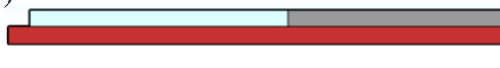

xenon lamp

(d)

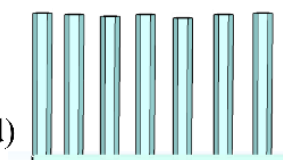

Figure 1. (a) Printing of silver electrodes and (b) ZnA layers on polyimide substrate. (c) Hydrothermal growth of $\mathrm{ZnO}$ nanowires and (d) photonic annealing of the nanowires.

\subsection{Electrochemical impedance spectroscopy (EIS)}

Electrochemical impedance measurements were carried out using Gamry (reference 600) potentiostat. As-grown and photonic annealed $\mathrm{ZnO}$ nanowires were introduced with $100 \mu \mathrm{L}$ PBS. Measurements were carried out at $10 \mathrm{mV}$ perturbation amplitude with reference to the measured open circuit potential in the frequency range of $1 \mathrm{~Hz}$ to $1 \mathrm{kHz}$. The impedance 
parameters were calculated by fitting using an equivalent circuit illustrated in Figure 3(b). The fitting errors were below $5 \%$.

\section{Results and Discussion}

SEM images of as-grown $\mathrm{ZnO}$ nanowires are shown in Figure S1. Figure 2a(i) and (ii) show SEM images of photonic annealed $\mathrm{ZnO}$ nanowires. The morphology of the nanowires was unchanged after photonic annealing. Figure S2 shows histograms on the diameter of asgrown and photonic annealed nanowires. The average length and diameter of as-grown nanowires were $545 \pm 94$ and $49 \pm 20 \mathrm{~nm}$, respectively. These were comparable to photonic annealed $\mathrm{ZnO}$ nanowires having average length and diameter of $534 \pm 101$ and $46 \pm 15 \mathrm{~nm}$, respectively.

EDX analysis was carried out on the $\mathrm{ZnO}$ nanowires to investigate its stoichiometry before and after photonic annealing. Figure 2(b) shows EDX spectrum of $\mathrm{ZnO}$ nanowires after photonic annealing. $\mathrm{Zn}$ and $\mathrm{O}$ peaks were from the $\mathrm{ZnO}$ nanowires, while the $\mathrm{Ag}$ peak originated from the silver electrodes. The stoichiometry of $\mathrm{ZnO}$ was almost 1:1 of zinc to oxygen, hence suggesting that photonic annealing did not affect the stoichiometry of the material.

X-ray diffraction (XRD) analysis of as-grown and photonic annealed nanowires is shown in Figure 2(c). Peaks from the substrate were observed between $20^{\circ}$ and $30^{\circ}$. $\mathrm{ZnO}$ peaks were observed at $31.7^{\circ}, 34.4^{\circ}, 36.2^{\circ}$ and $47.5^{\circ}$, which were associated with the hexagonal wurtzite phase of $\mathrm{ZnO}$ assigned as (100), (002), (101) and (102), respectively [7]. Diffraction peaks located at $38.1^{\circ}$ and $44.3^{\circ}$ can be indexed to (111) and (200) planes of printed silver electrodes, respectively [8]. The marginal increase in the intensity of $\mathrm{ZnO}(002)$ and $\mathrm{Ag}$ (111) peaks, after photonic annealing, is probably owing to a better orientation as suggested in previous reports [9]. 
Figure 2(d) shows photoluminescence (PL) spectra of as-grown and photonic annealed $\mathrm{ZnO}$ nanowires. Near band edge (NBE) and deep level emission (DLE) were observed from the spectra located at 376 and $600 \mathrm{~nm}$, respectively. After photonic annealing, the DLE peak was highly quenched indicating a reduction of defects in the material [1]. The NBE peak intensity was remarkably increased as a result of photonic annealing. After photonic annealing, the ratio of NBE to DLE of the photonic annealed nanowires was 0.82 , which was much larger than that of as-grown nanowires (e.g. 0.0064), suggesting a significant reduction in the defects.

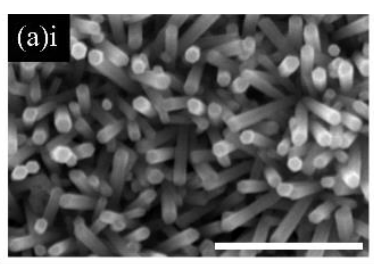

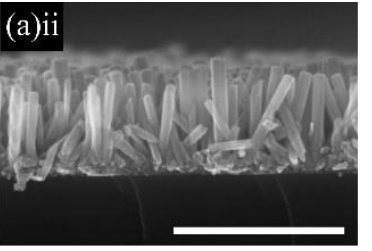

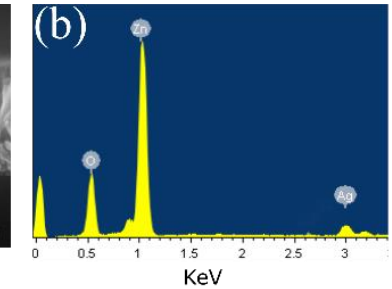

(c)

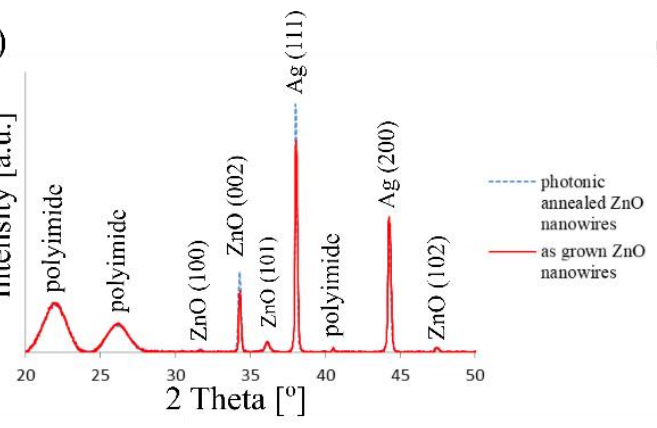

(d)

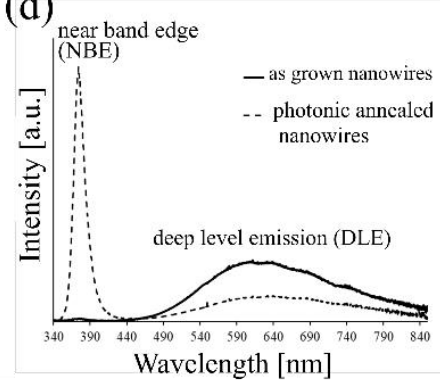

Figure 2. (a) SEM images showing (i) top and (ii) cross-sectional views of photonic annealed ZnO nanowires (scale bar is $500 \mathrm{~nm}$ ); (b) EDX analysis of $\mathrm{ZnO}$ nanowires grown on silver electrodes on polyimide substrate after photonic annealing; (c) XRD analysis of as-grown and photonic annealed ZnO nanowires; (d) PL spectra of as-grown and photonic annealed $\mathrm{ZnO}$ nanowires.

EIS measurement was performed to investigate the effect of photonic annealing on the impedance of $\mathrm{ZnO}$ nanowires. The EIS technique was previously used to determine the resistance of various materials [10, 11]. Figure 3(a) shows the Nyquist plot of as-grown and photonic annealed $\mathrm{ZnO}$ nanowires. It can be observed that the impedance of $\mathrm{ZnO}$ nanowires has drastically decreased after photonic annealing indicating better conductivity than asgrown nanowires. Hydrothermally grown $\mathrm{ZnO}$ nanowires could exhibit high density of surface defects due to absorption of oxygen molecules at the surface of nanowires. These 
defects would trap conduction electrons resulting in the formation of depletion layer near the surface of nanowires [1]. Photonic annealing would remove the absorbed oxygen molecules and reduce the depletion layer at the nanowires, thus leading to an enhancement in its electrical conductivity.

The impedance parameters of the nanowires (shown in Table 1) were obtained by fitting the EIS measurements using an equivalent circuit model as shown in Figure 3(b). The model reported here is similar to previously reported work by Jacobs et al [12]. Herein, the $\mathrm{ZnO}$ nanowires (in contact with PBS solution) were modelled as two parallel circuits connected to each other in series. The first circuit consisted of the $\mathrm{ZnO}$ resistance $\left(\mathrm{R}_{\mathrm{ZnO}}\right)$ and capacitance $\left(\mathrm{C}_{\mathrm{ZnO}}\right)$. The second circuit consisted of the charge transfer resistance $\left(\mathrm{R}_{\mathrm{ct}}\right)$ and the double layer capacitance $\left(\mathrm{C}_{\mathrm{dl}}\right)$ at the $\mathrm{ZnO} / \mathrm{PBS}$ interface. Since the PBS is not a redox probe, Rct was not considered in the simulation. Finally, Rs represents the resistance of PBS. The results showed that the resistance of $\mathrm{ZnO}$ nanowires has decreased by approximately six times after photonic annealing indicating an enhancement in the conductance of the nanowires. Furthermore, an increase in $\mathrm{C}_{\mathrm{dl}}$ by two orders of magnitude was observed after photonic annealing. The reduction of defects in $\mathrm{ZnO}$ nanowires that led to enhancement in the conductance of the nanomaterials would attract more ions to the $\mathrm{ZnO}$ surface and increase polarization of electrolyte counter ions at the $\mathrm{ZnO} / \mathrm{PBS}$ interface, thus increasing $\mathrm{C}_{\mathrm{dl}}$ at the interface. Such improvement in the electronic properties of $\mathrm{ZnO}$ nanowires is pivotal to its applications in biomedical, optical and energy storage etc. 
(a)

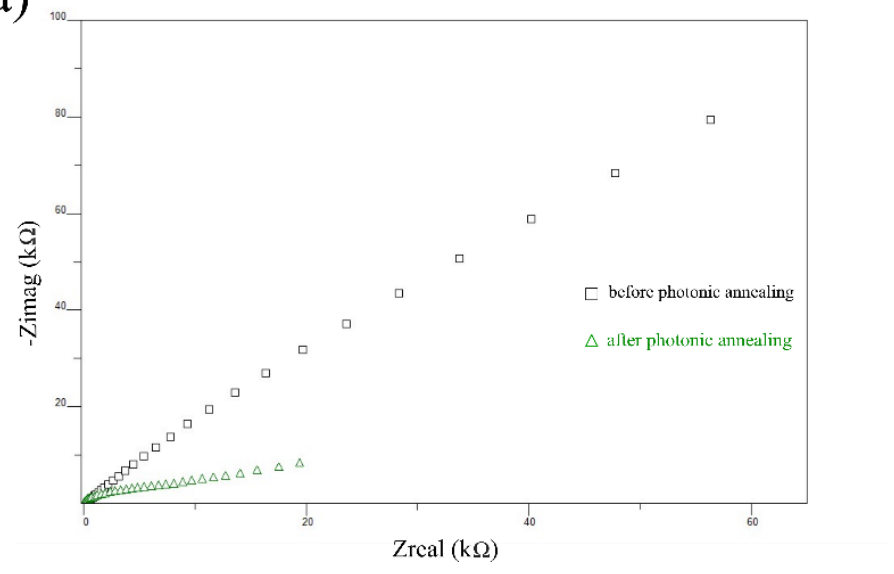

(b)

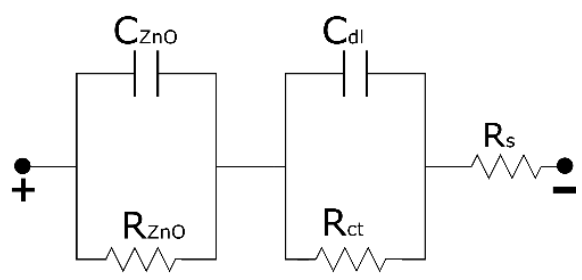

Figure 3. (a) Impedance spectra of $\mathrm{ZnO}$ nanowires before and after photonic annealing; (b) Simplified equivalent circuit.

Table 1. Impedance parameters of $\mathrm{ZnO}$ nanowires from EIS measurements.

\begin{tabular}{|c|c|c|}
\hline & $\begin{array}{c}\text { before photonic } \\
\text { annealing }\end{array}$ & $\begin{array}{c}\text { after photonic } \\
\text { annealing }\end{array}$ \\
\hline $\mathrm{R}_{\mathrm{ZnO}}(\Omega)$ & $8.9 \times 10^{3}$ & $1.53 \times 10^{3}$ \\
\hline $\mathrm{C}_{\mathrm{ZnO}}(\mathrm{F})$ & $3.26 \times 10^{-7}$ & $4.4 \times 10^{-6}$ \\
\hline $\mathrm{C}_{\mathrm{dl}}(\mathrm{F})$ & $8.48 \times 10^{-7}$ & $1.14 \times 10^{-5}$ \\
\hline $\mathrm{R}_{\mathrm{s}}(\Omega)$ & 49 & 48 \\
\hline
\end{tabular}

\section{Conclusion}

The results demonstrated an increase in the conductivity of hydrothermally grown $\mathrm{ZnO}$ nanowires due to the reduction of charge carrier traps after photonic annealing. Importantly, the annealing technique allows the use of organic substrates with low glass transition temperature and it has the potential to implement in series with roll-to-roll flexographic printing to facilitate low-cost mass production of high-performance flexible electronics.

\section{Acknowledgement}

This paper presents independent research funded by the National Institute for Health Research (NIHR) under its Invention for Innovation (i4i) Programme [Grant Reference Number II-LB-1117-20001]. The views expressed are those of the author(s) and not necessarily those of the NHS, the NIHR, or the Department of Health. 


\section{References}

[1] A. Kushwaha, M. Aslam, Defect induced high photocurrent in solution grown vertically aligned ZnO nanowire array films, Journal of Applied Physics 112(5) (2012) 054316.

[2] J. Maeng, S. Heo, G. Jo, M. Choe, S. Kim, H. Hwang, T. Lee, The effect of excimer laser annealing on ZnO nanowires and their field effect transistors, Nanotechnology 20(9) (2009) 095203.

[3] M. Hösel, F.C. Krebs, Large-scale roll-to-roll photonic sintering of flexo printed silver nanoparticle electrodes, Journal of Materials Chemistry 22(31) (2012) 15683-15688.

[4] E.B. Secor, B.Y. Ahn, T.Z. Gao, J.A. Lewis, M.C. Hersam, Rapid and Versatile Photonic Annealing of Graphene Inks for Flexible Printed Electronics, Advanced Materials 27(42) (2015) 6683-6688.

[5] K.A. Schroder, Mechanisms of Photonic Curing (TM): Processing High Temperature Films on Low Temperature Substrates, 2011.

[6] J.S. Lloyd, C.M. Fung, D. Deganello, R.J. Wang, T.G.G. Maffeis, S.P. Lau, K.S. Teng, Flexographic printing-assisted fabrication of ZnO nanowire devices, Nanotechnology 24(19) (2013) 195602.

[7] Z.M. Khoshhesab, M. Sarfaraz, M.A. Asadabad, Preparation of ZnO Nanostructures by Chemical Precipitation Method, Synthesis and Reactivity in Inorganic Metal-Organic and Nano-Metal Chemistry 41(7) (2011) 814-819.

[8] C.H. Liu, X.P. Yang, H.Y. Yuan, Z.D. Zhou, D. Xiao, Preparation of silver nanoparticle and its application to the determination of ct-DNA, Sensors 7(5) (2007) 708-718.

[9] G. Tai, ZnO nanowire arrays: controllable preparation and effect of annealing temperature on optical properties, Material Research Innovations 18 (2014) 20.

[10] F.E. Heakal, W.R. Abd-Ellatif, N.S. Tantawy, A.A. Taha, Characterization of electrodeposited undoped and doped thin $\mathrm{ZnO}$ passive films on zinc metal in alkaline $\mathrm{HCO}-/ \mathrm{CO} 2$ - buffer solution, Rsc Advances 8(69) (2018) 39321-39333.

[11] K. Habib, Surface Resistivity/Conductivity of Different Organic-Thin Films by a Combination of Optical Shearography and Electrochemical Impedance Spectroscopy, Optical Review 20(1) (2013) 5964.

[12] M. Jacobs, S. Muthukumar, A.P. Selvam, J.E. Craven, S. Prasad, Ultra-sensitive electrical immunoassay biosensors using nanotextured zinc oxide thin films on printed circuit board platforms, Biosensors \& Bioelectronics 55 (2014) 7-13. 